\title{
Pore-Scale Imaging and Analysis of Wettability Order, Trapping and Displacement in Three-Phase Flow in Porous Media with Various Wettabilities
}

\author{
Abdulla Alhosani $^{1}$ (D) $\cdot$ Branko Bijeljic $^{1} \cdot$ Martin J. Blunt ${ }^{1,2}$ (D)
}

Received: 19 October 2020 / Accepted: 6 April 2021 / Published online: 26 April 2021

(c) The Author(s) 2021

\begin{abstract}
Three-phase flow in porous media is encountered in many applications including subsurface carbon dioxide storage, enhanced oil recovery, groundwater remediation and the design of microfluidic devices. However, the pore-scale physics that controls three-phase flow under capillary dominated conditions is still not fully understood. Recent advances in three-dimensional pore-scale imaging have provided new insights into three-phase flow. Based on these findings, this paper describes the key pore-scale processes that control flow and trapping in a three-phase system, namely wettability order, spreading and wetting layers, and double/multiple displacement events. We show that in a porous medium containing water, oil and gas, the behaviour is controlled by wettability, which can either be water-wet, weakly oil-wet or strongly oil-wet, and by gas-oil miscibility. We provide evidence that, for the same wettability state, the three-phase pore-scale events are different under near-miscible conditions-where the gas-oil interfacial tension is $\leq 1 \mathrm{mN} / \mathrm{m}$ - compared to immiscible conditions. In a water-wet system, at immiscible conditions, water is the most-wetting phase residing in the corners of the pore space, gas is the most non-wetting phase occupying the centres, while oil is the intermediate-wet phase spreading in layers sandwiched between water and gas. This fluid configuration allows for double capillary trapping, which can result in more gas trapping than for two-phase flow. At near-miscible conditions, oil and gas appear to become neutrally wetting to each other, preventing oil from spreading in layers; instead, gas and oil compete to occupy the centre of the larger pores, while water remains connected in wetting layers in the corners. This allows for the rapid production of oil since it is no longer confined to movement in thin layers. In a weakly oil-wet system, at immiscible conditions, the wettability order is oil-water-gas, from most to least wetting, promoting capillary trapping of gas in the pore centres by oil and water during wateralternating-gas injection. This wettability order is altered under near-miscible conditions as gas becomes the intermediate-wet phase, spreading in layers between water in the centres and oil in the corners. This fluid configuration allows for a high oil recovery factor while restricting gas flow in the reservoir. Moreover, we show evidence of the predicted, but hitherto not reported, wettability order in strongly oil-wet systems at immiscible conditions, oil-gas-water, from most to least wetting. At these conditions, gas progresses through the pore space in disconnected clusters by double and multiple displacements; therefore, the injection of large amounts of water to disconnect the gas phase is unnecessary. We place
\end{abstract}

Extended author information available on the last page of the article 
the analysis in a practical context by discussing implications for carbon dioxide storage combined with enhanced oil recovery before suggesting topics for future work.

Keywords Three-phase flow $\cdot$ Porous media $\cdot \mathrm{X}$-ray imaging $\cdot$ Pore-scale $\cdot \mathrm{CO}_{2}$ storage . Enhanced oil recovery

\section{Introduction}

Unlocking the secrets to understanding three-phase flow-two liquids and a gas-in porous media is of utmost importance for many applications. Three-phase flow in porous structures is encountered during enhanced oil recovery (Lake 1989; Blunt et al. 1993), geological $\mathrm{CO}_{2}$ storage in oil and gas reservoirs (Qi et al. 2008; Melzer 2010, 2015a, b; Kramer 2020), environmental remediation of contaminated soils (Mayer and Miller 1993), as well as being indispensable in the design of microfluidic devices for food processing and drug delivery (Chen et al. 2019; Zúñiga and Aguilera 2008; Ferrara et al. 2009). However, despite these myriad applications we still do not have a complete predictive understanding of the phenomena that occur during three-phase flow (Alizadeh and Piri 2014). This is mainly attributed to the complex pore-scale physics that controls its behaviour (Blunt 2017).

In this work, we will first, briefly, describe the key pore-scale physical processes that control the fluid movement during three-phase flow, namely wettability order, spreading and wetting layers, and double/multiple displacement events (Blunt 2017). In a three-phase systemwhich in this paper, we will assume contains water, oil and gas - these processes are primarily impacted by: (i) surface wettability, which can either be water-wet, weakly oil-wet or strongly oil-wet and (ii) gas-oil miscibility (immiscible or near-miscible conditions) (Alhosani et al. 2019, 2020a; Scanziani et al. 2018a).

We will then provide a synthesized review of the latest insights into these processes achieved with three-dimensional pore-scale imaging (Alhosani et al. 2019, 2020a, b, 2021; Scanziani et al. 2018a, b, 2019, 2020; Blunt et al. 2021; Qin et al. 2019; Iglauer et al. 2013,2019; Feali et al. 2012). This technology has enabled in situ visualization of the pore space and the fluids within it, which has revolutionized our understanding of multiphase flow in porous media (Blunt et al. 2013; Wildenschild and Sheppard 2013; Brown et al. 2014; Bultreys et al. 2016; Cnudde and Boone 2013). The synthesis of this work is shown in Fig. 1, which summarizes the main findings of the three-phase flow X-ray imaging experiments listed in Table 1. These experiments were either performed using laboratory X-rays, which imaged fluid configurations at the end of a displacement, or synchrotron X-ray facilities, where images were acquired at approximately a time resolution of 1 min throughout a displacement.

The key features of three-phase flow, which will be the focus of discussion, are threefold: wettability order, wetting and spreading layers, and multiple displacements. These in turn control how each phase flows, its conductance, and how much is displaced or trapped.

\section{Wettability Order Controls Pore Occupancy}

In three-phase flow, it is wettability order that controls the arrangement of the fluid phases in the pore space (Blunt 2017); it determines which phase preferentially wets the solid surface and which one instead occupies the centre of the pores (defined here locally as wide regions of the pore space). The wettability order, from most to least wetting, can be 


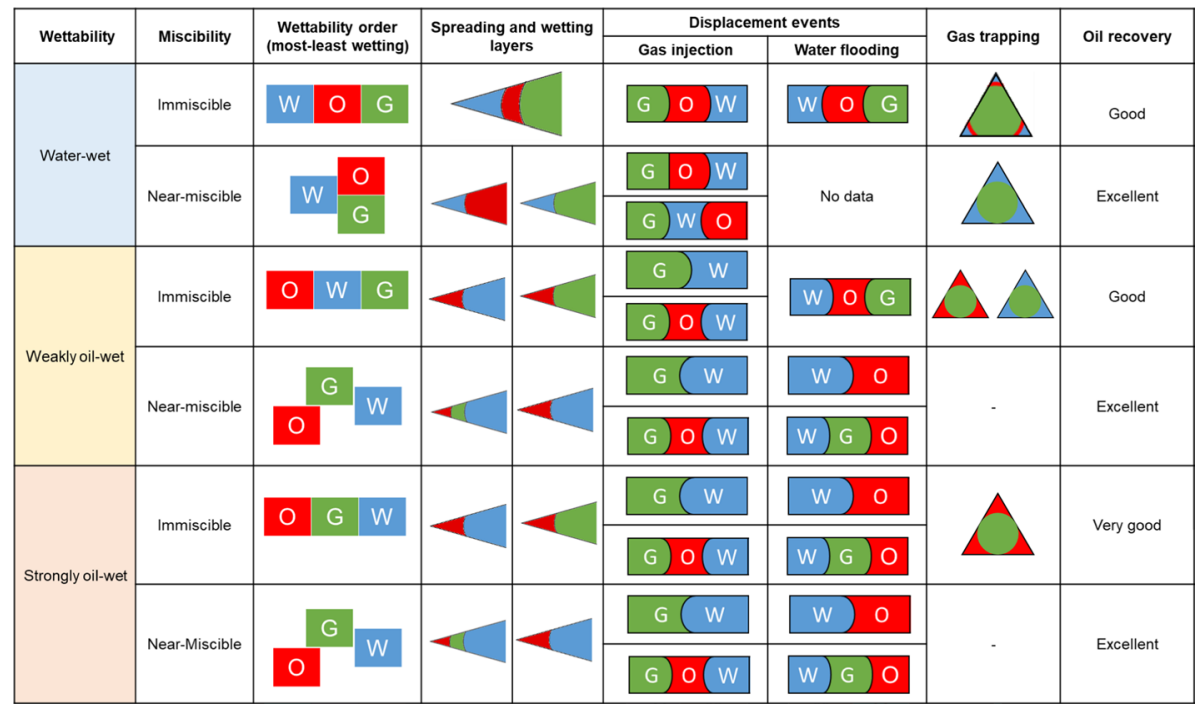

Fig. 1 The wettability orders, spreading and wetting layers, and double displacement events for various wettability and miscibility conditions during three-phase flow in porous media under capillary dominated conditions. The content summarizes the main findings of the three-phase flow pore-scale imaging studies listed in Table 1 (Alhosani et al. 2019, 2020a, b, 2021; Scanziani et al. 2018a, b, 2019, 2020; Qin et al. 2019; Iglauer et al. 2013, 2019; Feali et al. 2012)

defined either directly by the contact angle $\theta$ at the interface of each fluid pair, or indirectly through pore occupancy. Pore occupancy refers to the size of the pores occupied by each fluid phase. The most wetting phase tends to occupy the smallest pores and throats (restrictions in the pore space) as well as wetting layers. The most non-wetting phase preferentially resides in the centres of the larger pores, while the intermediate-wet phase occupies medium-sized pores and/or forms spreading layers sandwiched between the two other phases.

The arrangement of the phases in the pore space, determined by wettability order, has consequences for their flow conductivity and trapping. If a phase occupies large and/or intermediate-sized pores, it will have a larger flow potential compared to if it occupies smaller pores. However, this assumes that the phases are connected through the pore space. Capillary or residual trapping of a phase can occur when it exists as a disconnected bubble in the centre of the pores surrounded by another, more wetting, phase (Krevor et al. 2015; Andrew et al. 2014). Therefore, for a fluid phase to become trapped by another phase, in general, it must be less wetting than that phase. Hence, the most non-wetting phase has potentially the highest flow conductance when it is connected through the wide regions of the pore space but, conversely, can have the largest residual saturation if the flow pathways become blocked by more wetting phases. The wettability order is a function of surface wettability and gas-oil miscibility, as we show later (Blunt 2017; Alhosani et al. 2019, 2020a).

In this paper we will consider immiscible gas-oil systems, defined here when the gas-oil interfacial tensions are around $10 \mathrm{mN} / \mathrm{m}$ and the oil and gas phases have distinct physical properties. We will also study near-miscible systems with gas-oil interfacial tensions of around $1 \mathrm{mN} / \mathrm{m}$, which is an order of magnitude lower than the interfacial tension between the other fluid pairs. We will not discuss fully miscible gas injection, since at these conditions we see only two-phase flow (water and a hydrocarbon phase), or cases with 


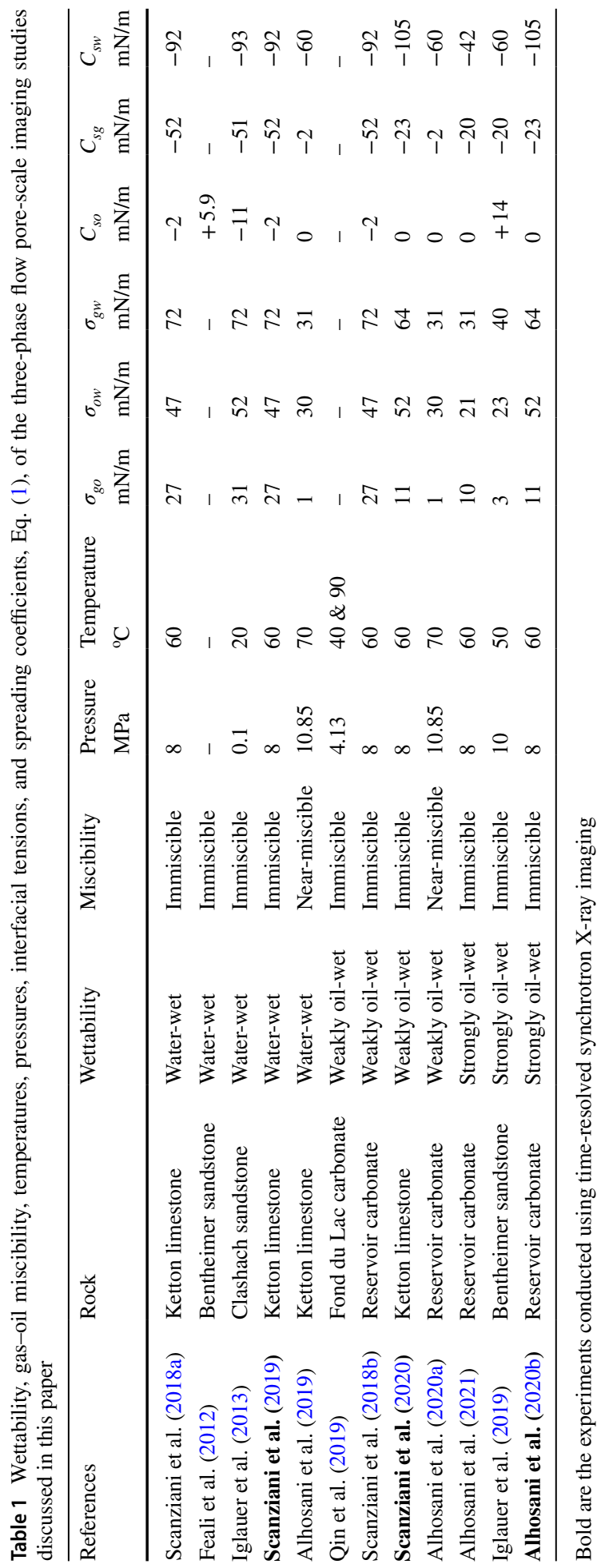


ultra-low gas-oil interfacial tensions where viscous effects become significant at the pore scale in comparison with capillary forces, as again the behaviour is often similar-in terms of recovery and trapping- to a miscible displacement (Lake 1989).

\section{Spreading and Wetting Layers Maintain Connectivity and Facilitate Trapping}

Fluid phases can form wetting and/or spreading layers in the pore space during three-phase flow (Hirasaki 1993). The formation of layers maintains the hydraulic connectivity of the phase in the pore space which allows it to flow at very low saturations, while facilitating the trapping of the other phases-while wetting layers can trap the other two phases, spreading layers can only trap the most non-wetting phase (Øren and Pinczewski 1994). The formation of wetting layers is dependent on the surface wettability, while the spreading coefficient $C_{s}$ of each fluid phase determines whether or not it will form spreading layers sandwiched between the two other phases in the pore space (Blunt 2017). The spreading coefficient of a phase $i$ can be found using the interfacial tensions, $\sigma$, between the three fluid phases ( $i, j$ and $k$ ) (Adamson and Gast 1967):

$$
C_{s i}=\sigma_{j k}-\sigma_{i j}-\sigma_{i k}
$$

Phase $i$ can spread in layers sandwiched between phases $j$ and $k$ if its equilibrium spreading coefficient is close to zero. If the spreading coefficient is strongly negative, the formation of spreading layers is not favoured. In Table 1, the values of spreading coefficient shown are measured at initial conditions before the three phases have reached mutual thermodynamic equilibrium. We assume that systems with an initial spreading coefficient that is positive or close to zero can form layers. In the text of the paper when we refer to the spreading coefficient, we refer to the equilibrium value.

\section{Multiple Displacements Allow Trapped Phases to be Displaced}

One unique characteristic of three-phase flow is double displacement which can occur under capillary dominated conditions (Øren and Pinczewski 1994; Øren et al. 1992; Fenwick and Blunt 1998). Double displacement refers to the displacement of one fluid phase by another, which in turn displaces another phase inside the porous medium (van Dijke and Sorbie 2003). Mathematically speaking, there are six possible double displacement events during three-phase flow: $(i-j-k),(i-k-j),(j-i-k),(j-k-i),(k-i-j),(k-j-i)$ (Blunt 2017). The dominant double displacement event depends on the injected phase, saturation history, wettability, miscibility, and spreading and wetting layers. Furthermore, it is also possible to have multiple displacements, with one phase displacing another and then another, with any number of intermediate steps (van Dijke and Sorbie 2003). Double and multiple displacements allow for both the mobilization of trapped fluid phases and their immobilization through displacement in wetting and spreading layers (Qin et al. 2019; Øren et al. 1992). Multiple displacement ensures that even in a capillary-controlled displacement, a phase can be displaced and advance through the pore space, even if it is surrounded by other phases (apparently trapped). 
The three-phase flow pore-scale processes that we have described-wettability order, layers and displacement events - have been extensively investigated using two-dimensional micromodels (Øren et al. 1992; Kantzas et al. 1988; Vizika and Lombard 1996; Sohrabi et al. 2000, 2001, 2004, 2008). However, the question remains whether or not the same pore-scale behaviour will persist in three-dimensional natural porous systems. It has been suggested that in natural systems it is not possible to strongly alter the wettability of the surface to the point where gas becomes the intermediate-wet phase (Lake 1989; Stone 1970,1973); it is generally assumed that gas will always remain as the most non-wetting phase. This is attributed to two reasons: (i) surface roughness, which retains water after primary drainage, may prevent the same strong wettability alteration as compared to micromodels where the surfaces are typically smooth (AlRatrout et al. 2018; Wenzel 1936; Cassie and Baxter 1944), and (ii) wettability in rocks is altered by being placed in contact with crude oil at high temperatures and pressures for long periods of time, whereas in micromodels a synthetic chemical is used that guarantees a uniform hydrophobic coating (Sohrabi et al. 2001; Salathiel 1973). Furthermore, the double/multiple displacements seen in micromodels, due to their two-dimensional structure, may occur less frequently in threedimensional media, where the pore space is more complex and better connected (Blunt 2017). We will demonstrate that neither of these assumptions are correct.

The emphasis of this work is on the three-dimensional pore-scale imaging of threephase flow in natural systems. Rather than providing a comprehensive review of experimental and modelling studies, we will instead focus our review on pore-scale imaging of three-phase flow in rock samples. We will answer the questions raised above pertaining to the use of two-dimensional micromodels to study three-phase flow, showing that it is possible to strongly alter the wettability of rocks, such that gas becomes the intermediate-wet phase and that double/multiple displacement events do occur in well-connected complex three-dimensional porous structures. Furthermore, we show that the three-phase pore-scale events are different under near-miscible gas-oil conditions compared to immiscible conditions, which is a phenomenon typically overlooked in three-phase flow studies. We then place the analysis in a practical context by discussing implications for flow and trapping before suggesting topics for future work.

\section{Mathematical and Physical Constraints}

Three-phase displacement is normally simplified by breaking down events into a series of two-phase processes. There are three combinations of two phases: oil-water $(o w)$, gas-water $(g w)$ and gas-oil ( $g o$ ), where the phases water, oil and gas are labelled $w, o$ and $g$, respectively. There are also three fluid-fluid interfacial tensions, three contact angles, three saturations, three interfacial curvatures and three capillary pressures.

The capillary pressure $P_{c i j}$ can be related to the interfacial curvature $\kappa_{i j}$ by the Young-Laplace equation (Armstrong et al. 2012):

$$
P_{c i j} \equiv P_{i}-P_{j}=\sigma_{i j} \kappa_{i j}
$$

for any combination of phases $i, j$ and $k$. The curvature $\kappa_{i j}$ is here defined as positive if phase $i$ bulges out into phase $j$ (and, as a consequence, phase $i$ is at a higher pressure than j).

However, there are constraints between the various three-phase parameters, as outlined below, so that only two contact angles, saturations and capillary pressures (or curvatures) 
are independent. The first constraint is easily derived from the definition of capillary pressure in Eq. (2) but is only valid for connected phases in capillary equilibrium:

$$
\sigma_{i k} \kappa_{i k}=\sigma_{i j} \kappa_{i j}+\sigma_{j k} \kappa_{j k}
$$

There is a similar constraint on contact angles, the Bartell-Osterhof relation (Bartell and Osterhof 1927; Blunt 2001) under the same assumptions:

$$
\sigma_{i k} \cos \theta_{i k}=\sigma_{i j} \cos \theta_{i j}+\sigma_{j k} \cos \theta_{j k}
$$

where $\theta_{i j}$ is a contact angle between phases $i$ and $j$ at the solid surface measured through (traditionally the denser) phase $j$.

There are two other constraints, inherent in the definition of curvature and contact angle:

$$
\kappa_{i j}=-\kappa_{j i}
$$

and

$$
\cos \theta_{i j}=-\cos \theta_{j i}
$$

\section{Determination of Contact Angle}

A key feature of three-phase flow and the analysis in this paper concerns wettability, or the local distribution of contact angles. Contact angle will be determined in two ways. The first - the geometric angle - is obtained by directly measuring the angle at the three-phase contact line between two fluid phases and the solid using an automatic approach (AlRatrout et al. 2017). This is a direct method from which a distribution of angles throughout the image can be found. However, the results could be inaccurate, due to uncertainties in the segmentation at the contact line. Furthermore, the angle measured represents the interfaces at rest, at the end of a displacement, rather than the angles that pertain when an interface moves through the pore space.

A second approach - to find the so-called thermodynamic contact angle-obtains the contact angle consistent with displacement by applying an energy balance from the difference between two images (Blunt et al. 2021,2019). If viscous dissipation is ignored, and assuming that all the energy input into the system ( $P d V$ work) is converted to surface energy, conservation of energy with application of the constraints, Eqs. (2)-(6), gives (Blunt et al. 2021):

$$
\left(\Delta a_{w s} \cos \theta_{o w}-\Delta a_{o w}-\phi \kappa_{o w} \Delta S_{w}\right) \sigma_{o w}=\left(\Delta a_{g s} \cos \theta_{g o}+\Delta a_{g o}-\phi \kappa_{g o} \Delta S_{g}\right) \sigma_{g o}+\Delta a_{g w} \sigma_{g w}
$$

where $s$ labels the solid, $a$ is the specific interfacial area (the area per unit volume), $\varphi$ is porosity, and $S$ is saturation. All the terms in Eq. (7) can be measured from pore-space images, except the contact angles. Since Eq. (7) is one equation for two unknown contact angles (the third is found from the Bartell-Osterhof relation, Eq. (4)), three images with two differences are needed for a unique determination $\theta_{o w}$ and $\theta_{g o}$. If a series of images have been acquired from synchrotron imaging, contact angles that best fit Eq. (7) for each difference between successive images can be found (Blunt et al. 2021).

The contact angles measured using both the geometric and thermodynamic approaches for the experiments discussed in the paper are listed in Table 2 and plotted in 
Table 2 Geometric and thermodynamic contact angle measurements between oil and water, gas and oil and gas and water at different conditions of wettability and miscibility

\begin{tabular}{llllll}
\hline Wettability & Miscibility & Method & $\theta_{o w}$ & $\theta_{g o}$ & $\theta_{g w}$ \\
\hline Water-wet & Immiscible & Geometric & $47^{\circ} \pm 5^{\circ}$ & $0^{\circ}$ & N/A \\
& & Thermodynamic & $48^{\circ} \pm 10^{\circ}$ & $0^{\circ}$ & $44^{\circ} \pm 10^{\circ}$ \\
& Near-Miscible & Geometric & $52^{\circ} \pm 22^{\circ}$ & $73^{\circ} \pm 17^{\circ}$ & $52^{\circ} \pm 18^{\circ}$ \\
& & Thermodynamic & - & - & - \\
Weakly oil-wet & Immiscible & Geometric & $105^{\circ} \pm 27^{\circ}$ & $57^{\circ} \pm 25^{\circ}$ & $80^{\circ} \pm 23^{\circ}$ \\
& & Thermodynamic & - & - & - \\
& Near-Miscible & Geometric & $112^{\circ} \pm 21^{\circ}$ & $67^{\circ} \pm 22^{\circ}$ & $108^{\circ} \pm 18^{\circ}$ \\
& & Thermodynamic & - & - & - \\
Strongly oil-wet & Immiscible & Geometric & $118^{\circ} \pm 25^{\circ}$ & $60^{\circ} \pm 24^{\circ}$ & $124^{\circ} \pm 24^{\circ}$ \\
& & Thermodynamic & $125^{\circ} \pm 10^{\circ}$ & $78^{\circ} \pm 10^{\circ}$ & $115^{\circ} \pm 10^{\circ}$ \\
\hline
\end{tabular}

The error in the geometric contact angle represents the standard deviation of the distribution, while in the case of the thermodynamic contact angle it indicates the uncertainty in the measurements. Data from (Alhosani et al. 2019, 2020a, b, 2021; Scanziani et al. 2018a, b, 2019, 2020; Blunt et al. 2021)

Fig. 2 Cosines of the geometric and thermodynamic gas-water and oil-water contact angles listed in Table 2. In orange are the angles measured in near-miscible gas-oil experiments, while in blue are the angles measured in immiscible experiments. The W-O-G, O-W-G, and O-G-W labels refer to the wettability order, from most to least wetting, in each quadrant, where $\mathrm{W}$ refers to water, $\mathrm{O}$ to oil, and $\mathrm{G}$ to gas

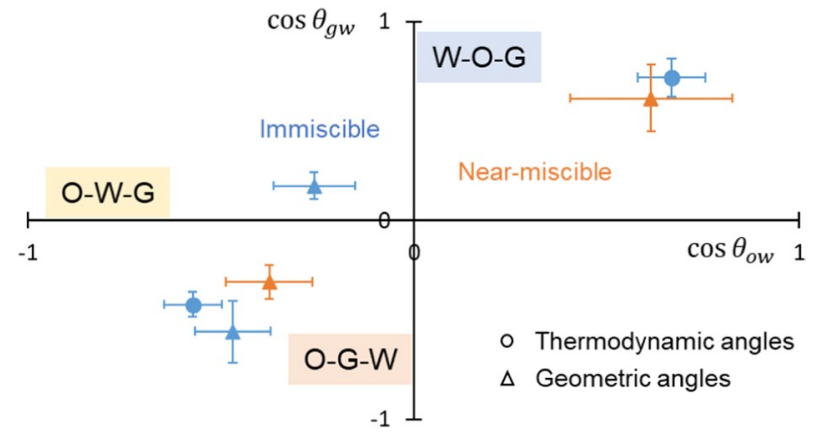

Fig. 2, where $\cos \theta_{g w}$ is plotted as a function of $\cos \theta_{o w}$. The quadrants of the graph define different wettability orders: for $\cos \theta_{o w}>0$ and $\cos \theta_{g w}>0$ the wetting order, from most to least wetting, is water-oil-gas; for $\cos \theta_{o w}<0$ and $\cos \theta_{g w}>0$ the order is oil-water-gas, while for $\cos \theta_{o w}<0$ and $\cos \theta_{g w}<0$ we have an oil-gas-water wetting order.

We will now discuss the main findings of the three-phase flow pore-scale imaging studies summarized in Fig. 1. We will examine the wettability order, spreading and wetting layers, and multiple displacement events at each surface wettability and miscibility condition, and discuss their implications for oil recovery and gas storage applications.

We will consider a displacement sequence that represents the situation typically encountered during enhanced oil recovery (EOR) processes: waterflooding to displace oil, followed by gas injection with subsequent water injection. Then, we use these porescale insights to suggest the optimum injection strategy to enhance the microscopic displacement efficiency for carbon storage and enhanced oil recovery projects. We will end with an overview of the difficulties and challenges that we need to overcome to gain a comprehensive understanding of three-phase flow in porous media, with a particular emphasis on the need to acquire high-quality three-phase relative permeability measurements. 


\section{Water-Wet Systems}

\subsection{Immiscible Conditions}

In a water-wet porous medium at immiscible gas-oil conditions, the wettability order is water-oil-gas from most to least wetting (Scanziani et al. 2018a, 2019; Iglauer et al. 2013; Feali et al. 2012). Water forms wetting layers occupying the corners, gas resides in the centres, while in most systems studied in the literature with fluids representative of subsurface conditions, oil spreads in layers sandwiched between gas and water, assuming a near-zero oil spreading coefficient, Eq. (1) (Scanziani et al. 2018a, 2019; Feali et al. 2012). An illustration of this wettability order in situ is shown in Fig. 3 (Scanziani et al. 2018a).

The wettability order can also be indirectly inferred from pore-scale images by performing a statistical analysis of pore occupancy to obtain a relation between the dimension of the pores and the phase sitting in its centre. Figure $3 \mathrm{~b}$ quantifies the pore occupancy after immiscible gas injection in a water-wet carbonate rock (Scanziani et al. 2018a). Water resides in the smallest pores, gas the biggest, while oil occupies intermediate size pores confirming the water-oil-gas, from most to least wetting, wettability order in the system.

This wettability order is in line with in situ measurements of fluid-fluid contact angles (see Table 2 and Fig. 2). The thermodynamic contact angles are $\theta_{o w}=48^{\circ} \pm 10^{\circ}, \theta_{g o}=0^{\circ}$, and $\theta_{g w}=44^{\circ} \pm 10^{\circ}$; this is consistent with the geometrically measured $\theta_{o w}=47^{\circ} \pm 5^{\circ}$ for a water-wet carbonate (Blunt et al. 2021). These results demonstrate that gas is non-wetting to both oil and water, oil is non-wetting to water and wetting to gas, while water is wetting to both oil and gas.

When gas is injected in a water-wet system after waterflooding, gas can only initially directly displace water before it contacts oil (Scanziani et al. 2019). As soon as gas contacts oil, oil spreads in layers between gas and water preventing their frequent direct contact in the pore space. Further displacement of water by gas is only possible through gas-oil-water double displacement. This type of displacement is known as
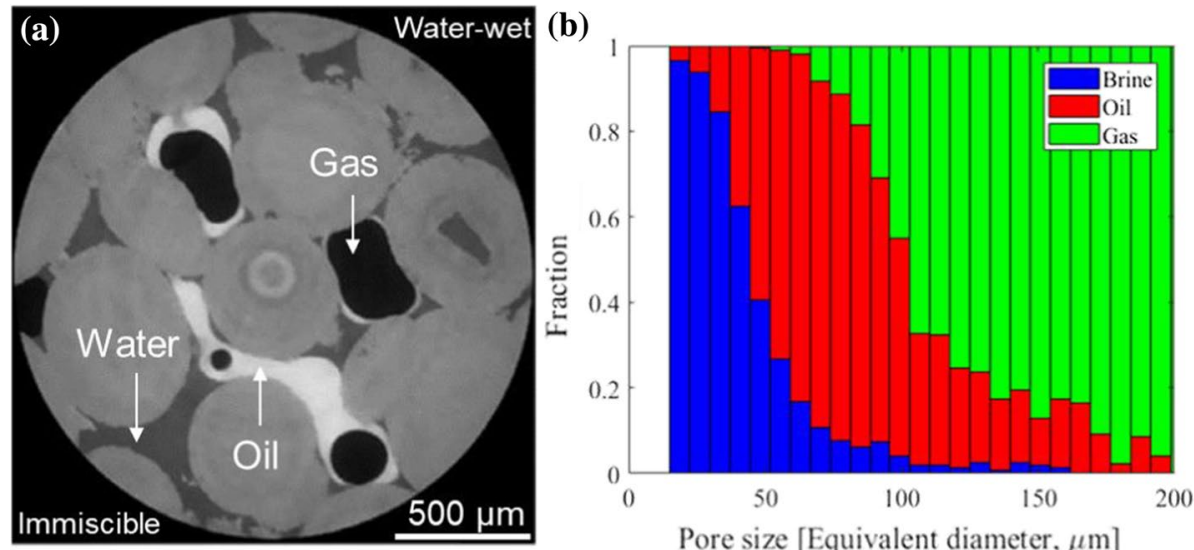

Pore size [Equivalent diameter, $\mu \mathrm{m}$ ]

Fig. 3 a A 2- $\mu$ m resolution two-dimensional raw pore-scale image showing the arrangement of gas, oil and water in the pore space of a water-wet carbonate rock at immiscible conditions. b A bar graph representing the pore occupancy of water, oil and gas in a water-wet rock after immiscible gas injection. In the greyscale image (a) the order from brightest to darkest is oil, rock, water, gas. In (b), gas is shown in green, oil in red, while water (brine) in blue. Data from Scanziani et al. (2018a) 
double drainage, which allows for the mobilization of trapped oil ganglia in the reservoir, increasing oil recovery (Øren et al. 1992), as seen by Scanziani et al. (2018a). They reported that tertiary gas injection, after waterflooding, allows for the production of up to $40 \%$ of the waterflood residual oil saturation (Scanziani et al. 2018a). Furthermore, additional oil can be recovered through drainage of oil layers by continuous gas injection, albeit very slowly (Scanziani et al. 2019).

During chase water re-injection, after gas injection, the main displacement process is water-oil-gas double displacement which is known as double imbibition. Water imbibes into the corners of the pore space through wetting layers; this causes the wetting layers to swell, disconnecting the oil layers surrounding gas, which results in capillary trapping of gas in the centre of the pores. Figure 4 shows an image of a trapped gas ganglion in the pore centre of a water-wet carbonate rock. Scanziani et al. (2018a) showed that, at immiscible conditions, $80 \%$ of the injected gas was trapped during chase water re-injection resulting in a residual gas saturation of $52 \%$ - the rest of the gas was displaced. The presence of spreading oil layers enhances the trapping of gas, since gas is strongly non-wetting to oil. This trapping mechanism-gas trapping by oil and oil trapping by water-is called double capillary trapping, which for gas storage applications can ensure that gas ganglia are immobilized by capillary and interfacial forces, especially as this gas is unlikely to dissolve in oil due to the immiscible conditions.
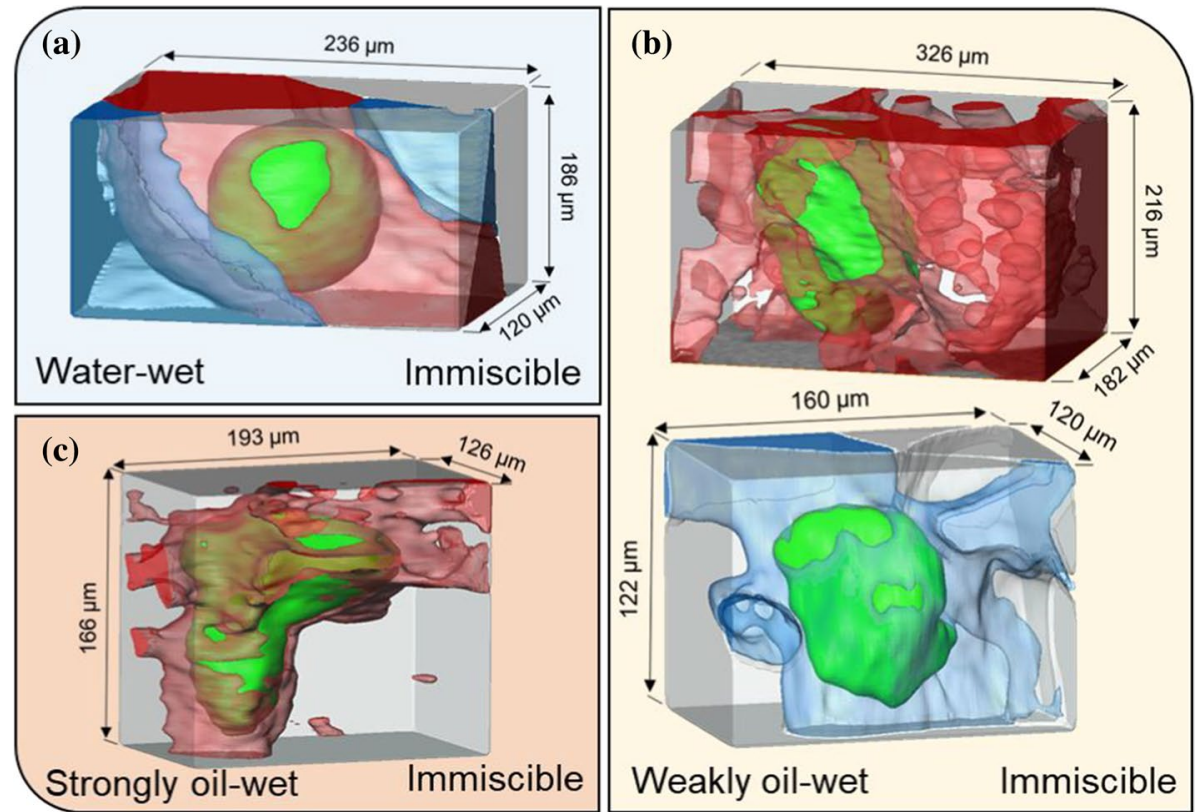

Fig. 4 Images of gas capillary trapping in a single pore of a carbonate rock at elevated temperatures and pressures for various surface wettabilities. Gas is shown in green, water in blue, oil in red, while the rock is rendered transparent. (a) In a water-wet rock, at immiscible conditions, gas gets double capillary trapped in the centre of large pores surrounded by oil spreading layers and water wetting layers (Scanziani et al. 2019). (b) In a weakly oil-wet rock, at immiscible conditions, gas is capillary trapped in the centre of the pore space by both oil and water (Scanziani et al. 2018b). (c) In a strongly oil-wet rock, at immiscible conditions, gas can only be trapped in the centre of the pore space by oil wetting layers (Alhosani et al. 2021). The pore-scale images were acquired with a resolution of $2 \mu \mathrm{m} /$ voxel size 
Moreover, chase water re-injection can further increase oil recovery in the reservoir as seen by Scanziani et al. (2018a), where the oil saturation decreased from 30 to $18 \%$ after water re-injection.

Under three-phase conditions, more gas can be trapped in the presence of both oil and water than in two-phase flow (for example, carbon dioxide storage in an aquifer). Rather than oil and gas competing to fill the larger pore spaces, oil in spreading layers is completely wetting to gas, which can result in more trapping than under two-phase conditions where the contact angle is not exactly zero (Blunt 2017; Scanziani et al. 2018a).

\subsection{Near-Miscible Conditions}

The injection of gas, in a water-wet system, at near-miscible conditions efficiently displaces oil out of the pore space with a microscopic displacement efficiency close to $100 \%$ (Alhosani et al. 2019). The low gas-oil interfacial tension, $\leq 1 \mathrm{mN} / \mathrm{m}$, results in a small capillary pressure between gas and oil making near-miscible conditions extremely favourable for oil recovery applications. This was experimentally observed by Alhosani et al. (2019), where the injection of gas in a water-wet rock at near-miscible conditions resulted in an oil recovery factor of $80 \%$, with the gas saturation reaching $76 \%$. However, subsequent waterflooding, after gas injection, was not performed in this experiment, and hence the amount of gas trapping is unknown (Alhosani et al. 2019). Nevertheless, as discussed below, with little remaining oil, the behaviour is likely to be similar to a two-phase gas-water system.

Alhosani et al. (2019) observed that at near-miscible conditions oil and $\mathrm{CO}_{2}$ became neutrally wetting to each other: the rock surface did not have a strong affinity to be preferentially coated by oil over gas. The strict wettability order, seen at immiscible conditions, where gas is distinctly more non-wetting than oil, breaks down as gas and oil compete to occupy the centre of the larger pores, while water remains connected in wetting layers in the corners of the pore space. This wettability order was observed directly from the pore-scale images (see Fig. 5). This was further confirmed by the measured fluid-fluid geometric contact angles (see Table 2) (the thermodynamic values have not been measured for this case), where oil and gas formed a large contact angle $\left(\theta_{g o}=73^{\circ}\right)$, indicating that they are almost neutrally wetting to the surface and had a similar contact angle with water $\left(\theta_{o w}=52^{\circ}\right.$ and $\theta_{g w}=52^{\circ}$ ) (Alhosani et al. 2019). In terms of pore occupancy, after gas injection, gas, oil and water occupied pores of all sizes (see Fig. 5b). The unexpected result is that water appears to be pushed into some of the larger pores-this is explained below as a consequence of double displacement processes.

The experimental observation of a gas-oil contact angle approaching $90^{\circ}$ as miscibility is reached (Alhosani et al. 2019) contradicts the empirical assumption of Sorbie and van Dijke (2010) and the experimental contact angle measurements of Al-Siyabi et al. (1999), where they observed a gas-oil contact angle that stays almost constant down to low gas-oil interfacial tensions, before jumping to zero as miscibility is reached. We attribute this distinct behaviour in the gas-oil contact angle to the fluids used in the investigations as Alhosani et al. (2019) studied a $\mathrm{CO}_{2}$-hydrocarbon system, while Al-Siyabi et al. (1999) performed the gas-oil contact angle measurements on a hydrocarbon-hydrocarbon system. Furthermore, in the case of Alhosani et al. (2019), the angle was measured in situ inside a three-dimensional porous rock in three-phase equilibrium, whereas Al-Siyabi et al. (1999) measured the gas-oil angle in a square capillary glass tube under two-phase equilibrium conditions. It is possible though that oil does indeed form layers in the in situ experiments and that the true contact angle with gas is close to zero, but that the layers are below the 

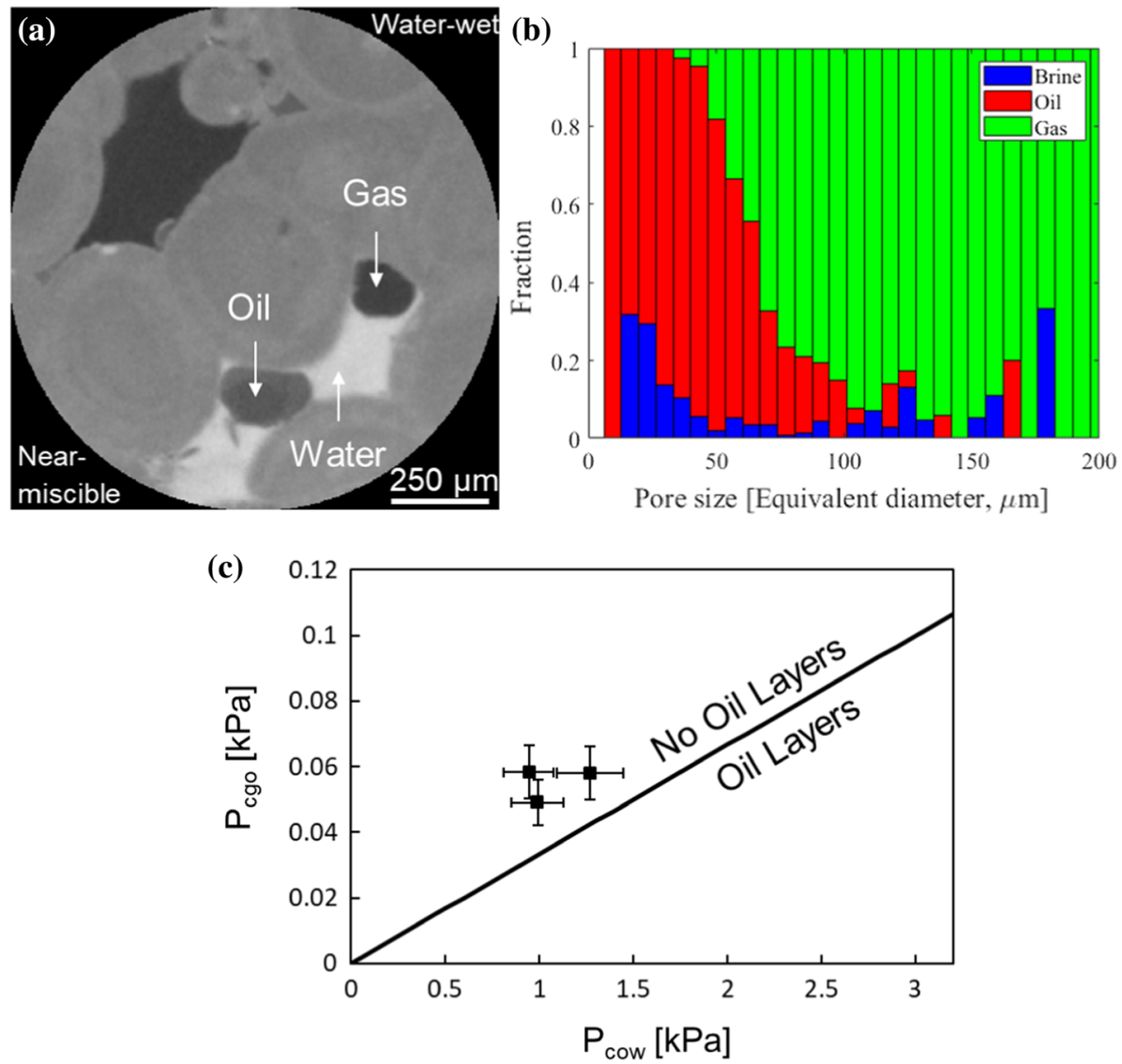

Fig. 5 a A 3.5- $\mu$ m resolution two-dimensional raw pore-scale image showing the arrangement of gas, oil and water in the pore space of a water-wet carbonate rock at near-miscible conditions. b A bar graph representing the pore occupancy of water, oil and gas in a water-wet rock after near-miscible gas injection. c An analysis of the capillary pressures to assess the formation of spreading oil layers at near-miscible conditions in a water-wet system. In the grey-scale image (a) the order from brightest to darkest is water, rock, oil, gas. In (b), gas is shown in green, oil in red, while water (brine) in blue. In (c), $P_{c}$ denotes the capillary pressure and subscripts $w, o$ and $g$ refer to water, oil and gas, respectively. Data from Alhosani et al. (2019)

resolution of the images; gas displaces the oil efficiently leaving either no, or very little, oil remaining in the pore space whose presence cannot be detected. Moreover, the images were acquired at the end of the displacement (Alhosani et al. 2019)—during gas injection there may be oil layers present with a near-zero effective contact angle between gas and oil. To sum up, it does remain an open question what the gas-oil contact angle is in the limit of a miscible system and whether or not this depends on the exact nature of the gaseous phase.

The other feature of near-miscible conditions is that oil is no longer confined to movement in spreading layers (oil does not form layers sandwiched between gas and water, since oil is no longer strongly wetting to gas) but instead flows rapidly in the centre of the pores; the residual oil saturation exists as disconnected clusters occupying the centre of the pore space rather than in layers, as seen under immiscible conditions. The absence of spreading 
oil layers can also be quantitatively assessed from a balance of the oil-water and gas-oil capillary pressures in the system. The capillary pressure analysis, shown in Fig. 5c, indicates that it is not possible for oil to spread in layers between gas and water in these experiments. Refer to Alhosani et al. (2019) for a detailed description of the methodology: a necessary geometric condition for the formation of layers in the pore space is that $\kappa_{o w}>\kappa_{g o}$, or, in terms of capillary pressure, Eq. (2) and Fig. 5c, $\sigma_{o w} P_{c g o}<\sigma_{g o} P_{c o w}$.

The absence of oil spreading layers allows gas to directly contact water in the pore space permitting the double displacement where gas displaces water that displaces oil. These double displacements allow water to enter some of the larger pores, as seen in Fig. 5b. Gas can remove water from some of the smaller pores, where it resides after oil injection; the water then has to shift to fill larger oil-filled elements through a local imbibition process. Nevertheless, since oil does not spread in layers surrounding gas, it is not possible to double capillary trap the gas phase during chase water re-injection; gas can only be capillary trapped by the water wetting layers. Therefore, in a water-wet system, we suggest that nearmiscible conditions are less favourable for gas storage compared to immiscible conditions.

To recap this section: near-miscible conditions are favourable for oil recovery, as expected, since gas directly displaces oil. More surprising is the impact of miscibility on spreading layers and trapping. In a water-wet medium, oil can form spreading layers under immiscible conditions which facilitates gas trapping by a double imbibition mechanism during tertiary waterflooding. Under near-miscible conditions, oil layers are not seen while gas and oil compete to occupy the largest pores, with direct contact of gas by water. If there is very favourable oil displacement from gas injection, the subsequent injection of water will simply be a gas-water displacement with similar trapping as seen in two-phase flow: there is no enhanced trapping of gas thanks to snap-off by oil spreading layers, as seen in immiscible systems.

\section{Weakly Oil-Wet Systems}

\subsection{Immiscible Conditions}

If a porous medium has undergone a severe wettability alteration, the system is referred to as strongly oil-wet; however, if its surfaces experience a moderate alteration in wettability, with oil-water contact angles $\theta_{o w}$ that are only slightly in excess of $90^{\circ}$, the porous medium is called weakly oil-wet (Blunt 2017). In weakly oil-wet systems, at immiscible conditions, the wettability order is oil-water-gas from most to least wetting (Scanziani et al. 2018b; Qin et al. 2019): this can be seen from the Bartell-Osterhof constraint, Eq. (4), where using the interfacial tension values, in Table 1, for immiscible conditions and assuming a gas-oil contact angle close to zero (oil is spreading) the contact angle between gas and water will be less than $90^{\circ},\left(\cos \theta_{g w}>0\right)$ even if $\cos \theta_{o w}<0$. This was experimentally confirmed by characterizing the geometric fluid-fluid contact angles at these conditions for a carbonate sample that had been in prolonged contact with crude oil, which gave $\theta_{o w}=105^{\circ}, \theta_{g o}=57^{\circ}$, and $\theta_{g w}=80^{\circ}$, as given in Table 2 (Scanziani et al. 2020).

Figures $4 \mathrm{~b}$ and 6 show pore-scale images of the distribution of oil, water and gas in the pore space of a weakly oil-wet rock at immiscible conditions (Scanziani et al. 2018b). Notice that gas bulges into water and water bulges into oil confirming that gas is the most non-wetting phase. This wettability order can further be inferred from the pore occupancy graph obtained by Scanziani et al. (2018b), where oil occupied the smallest pores, gas the 
biggest, while water occupied medium-sized pores (see Fig. 6b). The existence of gas in the biggest pores is consistent with the interpretation of weakly oil-wet core flood relative permeability measurements, where gas relative permeability is the highest and is independent of the water and oil saturations (Blunt 2017).

Water, the intermediate-wet phase, has a very large and negative spreading coefficient (see Table 1), which prevents it from spreading in layers sandwiched between gas and oil. This pore-scale arrangement allows gas to directly contact oil and water in the pore space permitting its trapping by both phases. This is illustrated in Fig. 4b, where capillary trapping of gas in the centres by oil and water was imaged in a weakly oil-wet reservoir rock. Scanziani et al. (2020) quantified the amount of residual gas trapped at these conditions after chase water re-injection and reported a gas saturation of $24 \%$.

However, the amount of gas trapping is lower than in a strongly water-wet rock of similar structure with a spreading oil. The gas is no longer strongly non-wetting to oil.

To summarize this section, the injection of gas in weakly oil-wet systems facilitates the flow of oil which resides in thick wetting layers. During gas injection, oil is displaced through gas-oil-water double displacement events. Gas can also directly displace water in the centres of the pore space, leaving oil connected in the corners. For oil recovery and gas storage applications, chase water re-injection is important as (i) it can significantly increase oil recovery through water-oil-gas double displacement and (ii) facilitate gas trapping by oil and water. Since gas is the most non-wetting phase, while connected, it can flow readily through the larger pores, which can result in excessive cycling of gas and insecure storage. Qin et al. (2019) demonstrated the importance of chase water re-injection for oil recovery by performing water-alternating-gas (WAG) injection in weakly oil-wet samples. The authors reported a residual oil saturation of almost $30 \%$ after gas injection, which dropped down to $20 \%$ after chase water re-injection.
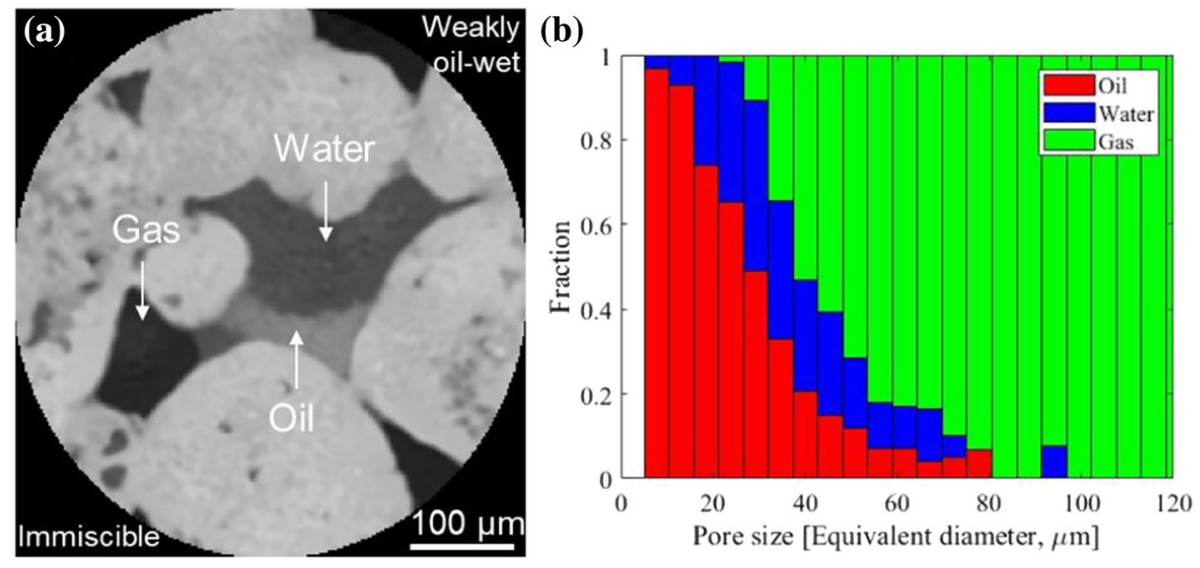

Fig. 6 a A 3.5- $\mu$ m resolution two-dimensional raw pore-scale image showing the arrangement of gas, oil and water in the pore space of a weakly oil-wet carbonate rock at immiscible conditions. (b) A bar graph representing the pore occupancy of water, oil and gas in a weakly oil-wet rock after immiscible gas injection. In the grey-scale image (a) the order from brightest to darkest is rock, oil, water, gas. In (b), gas is shown in green, oil in red, while water in blue. Data from Scanziani et al. (2018b) 


\subsection{Near-Miscible Conditions}

As gas and oil approach miscibility in a weakly oil-wet system, the wettability order is altered such that gas becomes unambiguously the intermediate-wet phase, water the most non-wetting phase, while oil remains the most wetting phase (Alhosani et al. 2020a). This can be seen from the Bartell-Osterhof relationship, Eq. (4): when $\sigma_{g o}$ tends to zero, we find $\sigma_{g w} \sim \sigma_{o w}$ (since oil and gas are similar - see Table 1) and hence $\cos \theta_{g w} \sim \cos \theta_{o w}$. Therefore, even if the system is only weakly oil-wet, gas will also be wetting (albeit also weakly) to water as it has similar properties to oil. This can be seen in Table 2, where the measured geometric contact angles between oil and water and gas and water were $112^{\circ}$ and $108^{\circ}$, respectively, at these conditions for a carbonate rock that had been in contact with crude oil.

If the gas-oil contact angle, $\theta_{g o}$, remains constant as the wettability-defined by the value of $\theta_{o w}$-is varied, then from the Bartell-Osterhof relationship, Eq. (4), $\cos \theta_{g w}$ should be linearly related to $\cos \theta_{\text {ow }}$ (van Dijke and Sorbie 2003; Sorbie and van Dijke 2010). Using this linear relationship, the transition from gas being the most non-wetting phase in an immiscible system to water becoming most non-wetting at near-miscible conditions was first predicted by van Dijke and Sorbie (2003). This was later experimentally verified by Grate et al. (2012) by measuring fluid-fluid contact angles on a flat surface using the static sessile drop method. While-as we show in Fig. 2-there is not an obvious linear relationship between the cosines of the gas-water and oil-water contact angles, we do see a shift towards gas being more wetting to water as we lower the gas-oil interfacial tension. Nonetheless, we do not see the predicted linear trend because $\theta_{g o}$ also changes with wettability. In addition, the Bartell-Osterhof relationship holds for contact angles measured at the same location in three-phase equilibrium. In our experiments, we measure contact angles between pairs of phases at different locations which means that Eq. (4) may not be strictly applicable to our measurements.

This wettability order-oil-gas-water, from most to least wetting-is typically associated with strongly oil-wet systems, where for immiscible conditions the Bartell-Osterhof Eq. (4) gives $\cos \theta_{g w}<0$ only if $\cos \theta_{o w} \sim-1$; however, using pore-scale imaging we can see that gas becomes wetting to water in weakly oil-wet systems under near-miscible conditions (Alhosani et al. 2020a) (see Fig. 7). Performing the pore occupancy analysis on pore-scale images of a weakly oil-wet rock at near-miscible conditions indicates that oil resides in the smallest pores, water the biggest, while gas occupies the mediumsized pores (Fig. 7b). This is consistent with the observed reduction in the gas relative permeability under weakly oil-wet near-miscible conditions (Fatemi and Sohrabi 2013); this behaviour can now be explained using the wettability order.

This again confirms that although the surface wettability is the same, the pore-scale behaviour of fluids is different under near-miscible and immiscible conditions, as also seen in water-wet systems and described in Sect. 2.

Since gas is more wetting to the surface than water, it cannot be capillary trapped by water in the centres of the pore space. This may have an adverse impact on the security of gas storage applications. Furthermore, at these conditions, gas is present in spreading layers sandwiched between water and oil due to its spreading coefficient being close to zero, as given in Table 1. Figure 8 shows evidence of the existence of gas in layers at near-miscible weakly oil-wet conditions. The gas phase is disconnected except for thin layers of low flow conductivity which limits its mobility in the pore space making it difficult—but not impossible — for the gas to escape, and thus providing an alternative 

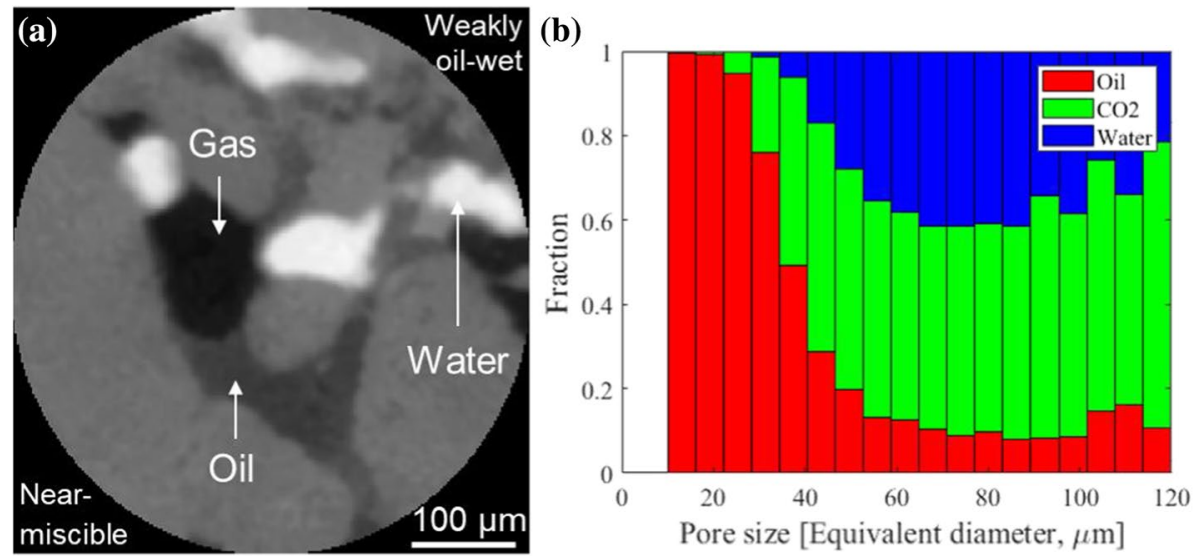

Fig. 7 a A 3.5- $\mu$ m resolution two-dimensional raw pore-scale image showing the arrangement of gas, oil and water in the pore space of a weakly oil-wet carbonate rock at near-miscible conditions. $\mathbf{b}$ A bar graph representing the pore occupancy of water, oil and gas in a weakly oil-wet rock after near-miscible gas injection. In the grey-scale image (a) the order from brightest to darkest is water, rock, oil, gas. In (b), gas $\left(\mathrm{CO}_{2}\right)$ is shown in green, oil in red, while water in blue. Data from Alhosani et al. (2020a)

gas storage mechanism. Using direct numerical simulation, it was seen that the $\mathrm{CO}_{2}$ conductance was reduced by a factor of 10 compared to an equivalent situation where it occupied the larger pores (Alhosani et al. 2020a). If gas is considered safely stored upon injection only, due to its limited mobility, this mitigates the need for chase water reinjection that reduces the gas storage capacity in the reservoir. This was demonstrated

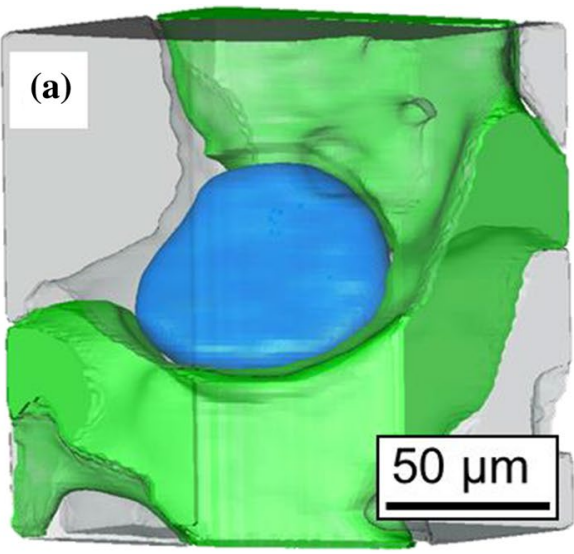

Weakly oil-wet Near-miscible

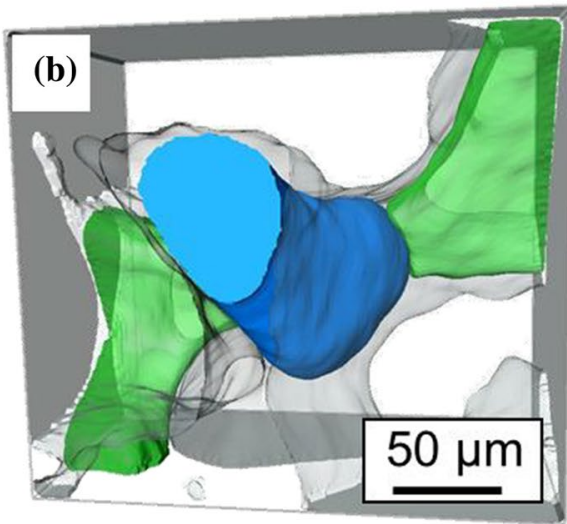

Strongly oil-wet Immiscible

Fig. 8 Three-dimensional images of the configuration of gas and water in a single pore at a near-miscible weakly oil-wet conditions and $\mathbf{b}$ immiscible strongly oil-wet conditions. Gas is shown in green, while water is blue. Gas spreads in layers at near-miscible conditions, while it exists as disconnected clusters at immiscible conditions. The size of the three-dimensional subvolumes shown at immiscible and near-miscible conditions are $185 \times 209 \times 121 \mu^{3}$ and $109 \times 134 \times 152 \mu \mathrm{m}^{3}$, respectively. The pore-scale images were acquired at a resolution of $1.82 \mu \mathrm{m} /$ voxel size. From Alhosani et al. (2021) 
by Alhosani et al. (2020a) as they showed that the stored gas saturation in a reservoir rock decreased from 33 to $21 \%$ after water re-injection. However, although spreading gas layers restrict flow, they can prevent the oil wetting layers from surrounding gas in the pore space disallowing capillary trapping of gas by oil.

Gas injection at these conditions is especially favourable for oil recovery applications. This was confirmed by Alhosani et al. (2020a), who reported an increase of up $30 \%$ in the oil recovery factor by near-miscible gas injection and another $33 \%$ by the subsequent water injection. The significant increase in oil recovery is attributed to two reasons: (i) the low gas-oil capillary pressure which results in a very efficient displacement of oil by gas, and (ii) during gas injection, gas, the intermediate-wet phase, displaces oil in the corners of the pore space, leaving water stranded in the centres which increases oil recovery and limits water production.

Two types of displacement occur when gas is injected at these conditions: gas-oil-water double displacement, and gas-water direct displacement. During chase water re-injection, water can displace oil directly in the pore space, or displace gas that further displaces oil out of the small sized pores.

To recap, as in a water-wet system, near-miscible gas injection is favourable for oil recovery. The miscibility also changes the wetting order in weakly oil-wet systems, unlike in a water-wet system: while oil remains the most wetting phase, when gas and oil are immiscible, gas is generally the most non-wetting phase, filling the largest pores, while gas becomes intermediate-wet under near-miscible conditions and forms spreading layers. Note the difference with the water-wet case, where no layers of the intermediate phase (oil) were seen under near-miscible conditions. When gas is the most non-wetting phase, tertiary water injection is needed to trap the gas effectively; instead, as an intermediate phase, the gas mobility is restricted and may be stored effectively after gas injection without the need to re-inject water.

\section{Strongly Oil-Wet Systems}

\subsection{Immiscible Conditions}

For many years, it was believed that the theoretically predicted wettability order for strongly oil-wet systems-oil-gas-water from most to least wetting-does not occur in natural porous media. However, this wettability order is possible based on the Bartell-Osterhof relation, Eq. (4), as discussed previously, when $\cos \theta_{o w} \sim-1$ (van Dijke and Sorbie 2002) and has been seen in micro-model experiments (Sohrabi et al. 2001). In a recent study (Alhosani et al. 2021) the authors successfully altered the wettability of a reservoir rock towards strongly oil-wet conditions and visualized, using pore-scale imaging, the hypothesized wettability order. To further confirm the wetting order in the system, the authors quantified the in situ fluid-fluid contact angles (see Table 2), which demonstrated that oil is wetting to both water and gas, gas is non-wetting to oil and wetting to water, while water is non-wetting to both oil and gas (Alhosani et al. 2021): this was the case for both the geometric and thermodynamic estimates of contact angles (Alhosani et al. 2020b). Moreover, the pore occupancy statistics indicated that, on average, oil resides in the smallest pores and water the biggest, while gas occupies intermediate-sized pores (see Fig. 9).

Although the wettability order under strongly oil-wet immiscible conditions is the same as at weakly oil-wet near-miscible conditions, the pore-scale fluid configuration is 
different. While gas spreads in layers at near-miscible conditions, at immiscible conditions, gas exists in the pore space as disconnected ganglia (see Fig. 8b); gas, the intermediate-wet phase, does not spread in layers since it has a large and negative spreading coefficient (see Table 1). The existence of gas in disconnected clusters allows for the capillary trapping of gas by oil in the centre of the pores (see Fig. 4c). However, capillary trapping of gas by water is still not possible since gas is more wetting to the surface than water.

When gas is injected at immiscible conditions, it progresses through the pore space in disconnected clusters by gas-oil-water double and multiple displacements, combined with gas-water direct displacement. This was first observed by Alhosani et al. (2020b), who used time-resolved synchrotron imaging to capture the connectivity of gas during gas injection in a strongly oil-wet reservoir rock at immiscible conditions. Figure 10 shows the connectivity of the gas phase during gas invasion at different time-steps-each colour represents a different gas cluster. The authors attributed this behaviour to the pore-scale events that govern the gas movement in the porous medium, which they termed three-phase Haines jumps. They observed that as gas displaces either oil or water, it rapidly progresses to fill several pores which causes it to retract from regions further away from the gas front to enable this fast filling. This retraction leads to a permanent disconnection of gas ganglia which fail to get reconnected as gas injection proceeds. While this is not seen in twophase flow, the presence of double and multiple displacement mechanisms allows the gas to progress through the pore space while remaining discontinuous even under capillarycontrolled conditions. The disconnected gas ganglia reach a new position of capillary equilibrium in the pore space and can only be displaced through double/multiple displacement events (Alhosani et al. 2020b).

This type of advance is particularly favourable for gas storage applications since gas gets disconnected upon injection; the injection of large amounts of water to trap the gas is unnecessary. Therefore, gas injection alone should provide a favourable storage capacity. Alhosani et al. (2021) showed that the gas saturation can reach up to $25 \%$ in a strongly oilwet rock at immiscible conditions.

However, the injection of water may be desirable to increase the oil recovery as the microscopic displacement efficiency of oil by gas is reduced under immiscible conditions due to the high gas-oil interfacial tension; gas injection only recovered $16 \%$ of

Fig. 9 A bar graph representing the pore occupancy of water, oil and gas in a strongly oil-wet rock after immiscible gas injection. Gas $\left(\mathrm{CO}_{2}\right)$ is shown in green, oil in red, while water in blue. Data from Alhosani et al. (2021)

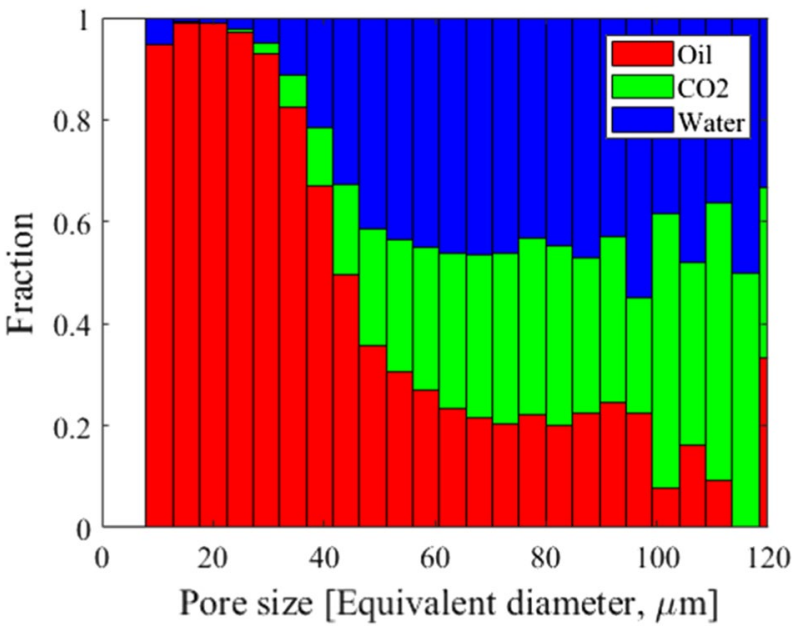



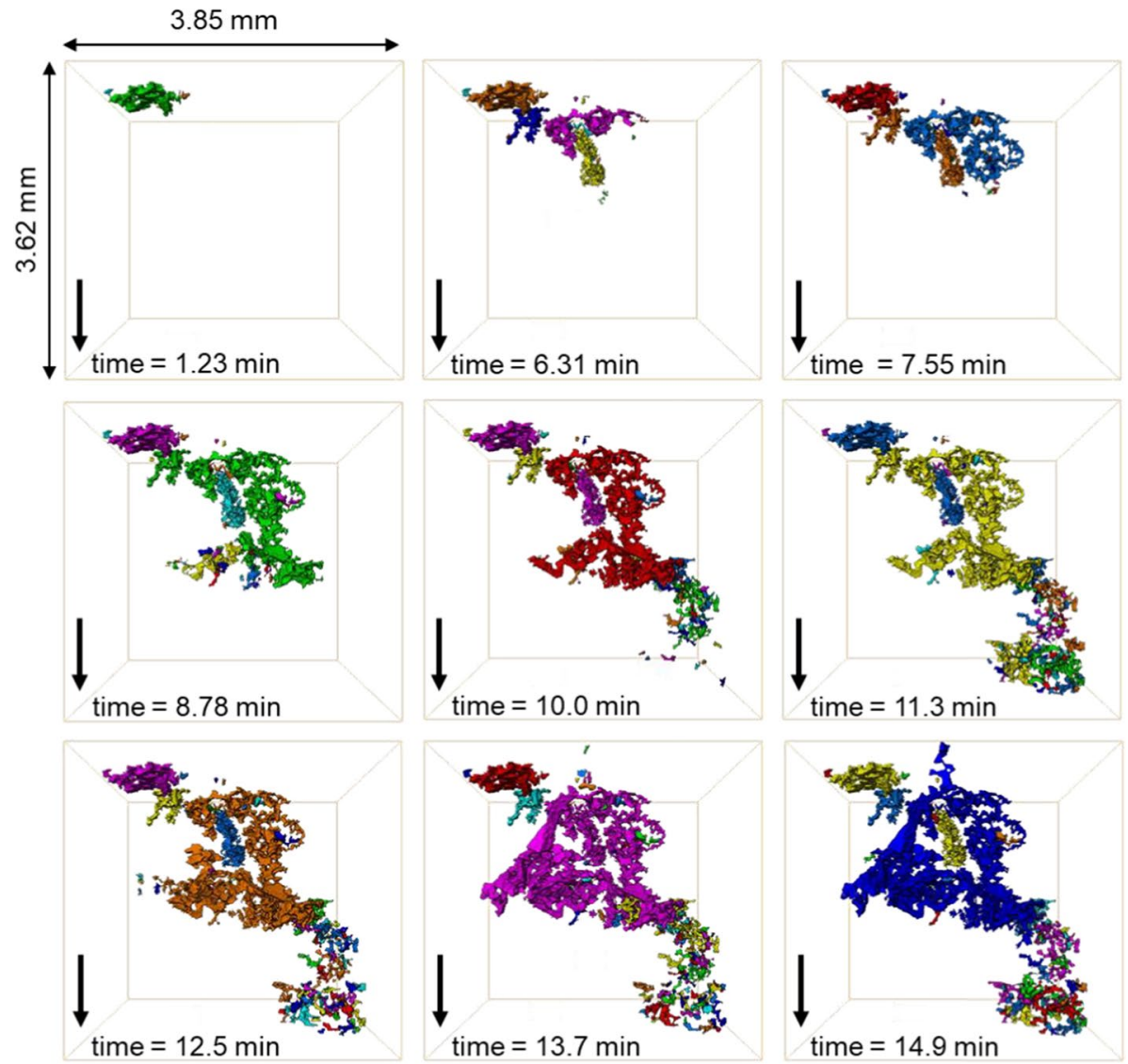

Fig. 10 Three-dimensional maps of the gas phase showing the evolution of gas connectivity during gas injection in a strongly oil-wet reservoir rock at immiscible conditions. Each colour represents a disconnected gas cluster. The black arrow points towards the direction of flow. Data from Alhosani et al. (2020b)

the oil in place; by re-injecting water, after gas injection, an additional $31 \%$ of oil was recovered (Alhosani et al. 2021). During chase water re-injection, two types of displacement can occur: (i) water-oil direct displacement and (ii) water-gas-oil double displacement.

\subsection{Near-Miscible Conditions}

No pore-scale imaging studies have been reported at near-miscible conditions in strongly oil-wet systems; however, we presume that the wettability order, wetting and spreading layers, and double displacement events will be similar to those observed under weakly oil-wet near-miscible conditions. In this case, the local displacement efficiency of oil will be favourable, with restricted flow of the gas in spreading layers. 


\subsection{CCS-EOR Implications}

Carbon dioxide capture and storage (CCS) has been recognized as an imperative technology for mitigating climate change (Bickle 2009; Scott et al. 2013). To meet the climate change targets set by the Intergovernmental Panel on Climate Change (IPCC), the underground storage of up to a thousand gigatonnes of $\mathrm{CO}_{2}$ is required by the end of this century (Metz et al. 2005; Allen et al. 2019). The initial rapid deployment of largescale $\mathrm{CO}_{2}$ sequestration is considered to be most feasible in depleted oil and gas reservoirs (Brownsort 2015; Melzer 2010; Kramer 2020). This is attributed to many reasons including the: (i) large secure storage capacity of hydrocarbon reservoirs; (ii) geological knowledge of the reservoirs; (iii) built infrastructure and transportation routes; and (iv) a financial incentive from enhanced oil recovery (EOR).

In this section, we will suggest the optimum $\mathrm{CO}_{2}$ injection strategy during CCS-EOR to improve the microscopic displacement efficiency in oil reservoirs under various wettability and miscibility conditions. Our discussion is limited to enhancing the microscopic displacement efficiency and does not consider the large-scale sweep efficiency and operational costs. We confine our discussion to immiscible and near-miscible conditions, but not fully miscible conditions, where oil and gas flow together as one phase in the reservoir. Although miscible conditions are favourable for local displacement efficiency, the high mobility contrast between the injected gas and resident oil can lead to channelling, while the hydrocarbon phase maintains a high mobility, which facilitates the recycling of $\mathrm{CO}_{2}$ through the reservoir (Lake 1989), and so is generally less favoured for secure storage. To optimize $\mathrm{CO}_{2}$ injection during CCS-EOR, the goal is to store as much $\mathrm{CO}_{2}$ as possible while maximizing oil production.

To optimize CCS-EOR in a water-wet oil reservoir, at immiscible conditions, we suggest a water-alternating-gas (WAG) injection strategy. When $\mathrm{CO}_{2}$ is injected, it remains connected through the largest pores which allows it to flow rapidly in the pore space and potentially escape through any abandoned boreholes. Therefore, the injection of water is necessary to double capillary trap $\mathrm{CO}_{2}$, as shown in Fig. 4a, immobilizing it in the pore space of the reservoir. Indeed the amount of trapping can be larger than under equivalent two-phase conditions, since spreading oil is completely wetting to the gas. Furthermore, the injection of water can directly displace oil in the pore space, boosting oil recovery and facilitating drainage of oil through spreading layers.

Although double capillary trapping of $\mathrm{CO}_{2}$ is not possible at near-miscible conditions, in a water-wet system, WAG is still the favourable injection strategy. The injection of water, after $\mathrm{CO}_{2}$, at near-miscible conditions allows $\mathrm{CO}_{2}$ to become trapped in the pore centres by water wetting layers, securing $\mathrm{CO}_{2}$ storage. Nevertheless, it is recommended that $\mathrm{CO}_{2}$ is injected alone at the beginning to maximize $\mathrm{CO}_{2}$-oil contact in the reservoir, recovering large amounts of oil due to the high microscopic displacement efficiency of oil by $\mathrm{CO}_{2}$.

The selected injection strategy is more pertinent in weakly oil-wet reservoirs, where $\mathrm{CO}_{2}$ can go from residing in the centre of the pores to spreading in layers by switching from immiscible to near-miscible conditions. At immiscible conditions, the lower gas-oil displacement efficiency and the possibility of capillary trapping $\mathrm{CO}_{2}$ in the centres by both oil and water (see Fig. 4b), suggest that WAG is the optimum injection strategy. On the other hand, at near-miscible conditions, where $\mathrm{CO}_{2}$ is confined to movement in low mobility spreading layers, continuous $\mathrm{CO}_{2}$ injection is recommended for two reasons: (i) $\mathrm{CO}_{2}$ can efficiently displace oil in the corners of the pore space 
significantly increasing oil recovery, and (ii) the injected $\mathrm{CO}_{2}$ has very low flow conductivity in the reservoir which makes it highly improbable-but not impossible-for the stored $\mathrm{CO}_{2}$ to flow towards abandoned wells and escape. Furthermore, an injection strategy of $\mathrm{CO}_{2}$ injection only can maximize the $\mathrm{CO}_{2}$ storage capacity in the reservoir as a lower fraction of the pore space is then occupied by water.

It is more difficult to predict the optimum injection strategy in strongly oil-wet reservoirs at immiscible conditions based on microscopic displacement efficiency insights only. At these conditions, $\mathrm{CO}_{2}$ is disconnected upon injection, hence, an injection strategy of $\mathrm{CO}_{2}$ injection only will produce oil while maximizing the storage capacity since the $\mathrm{CO}_{2}$ remains poorly connected in the pore space. Nevertheless, at these conditions, the gas-oil displacement efficiency is reduced, and in some cases water injection may be necessary to boost oil recovery. Moreover, the injection of water at these conditions can promote capillary trapping of $\mathrm{CO}_{2}$ by oil in the pore centres (see Fig. 4c). Therefore, there is an inevitable trade-off between storage capacity and oil recovery during the design of CCS-EOR in strongly oil-wet reservoirs at immiscible conditions. Additional knowledge, including the residual oil saturation and water availability, may be needed to select the optimum injection strategy.

\subsection{Challenges and Future Work}

This paper has highlighted the recent insights into three-phase flow in porous media gained using three-dimensional pore-scale X-ray imaging. We provide in situ evidence of the different wettability orders, spreading and wetting layers, and double displacements at various conditions and discuss their impact on flow and trapping during the simultaneous flow of three fluid phases. We demonstrate that these pore-scale events are a function of wettability and miscibility.

This work has focussed on studying three-phase flow in porous rocks only (Alhosani et al. 2019, b, 2020, 2021; Scanziani et al. 2018a, b, 2019, 2020), whereas the pore-scale behaviour of fluids may vary in different porous media, e.g. microfluidic devices, shales or fractured rocks (Chen et al. 2019; Zhao 2013; Sabti et al. 2019; Sayed et al. 2019). The impact of using live oils on gas-oil miscibility must also be investigated since all the threephase flow pore-scale imaging studies discussed used dead oils (that is oil with no dissolved natural gas at reservoir conditions). The scope of this study was confined to gas-oil-water three-phase systems; however, the use of pore-scale imaging can be extended to investigate the flow of liquid-liquid-solid and gas-liquid-solid systems which occur in chemical engineering applications (Kundu et al. 2008; Falcone et al. 2009). Furthermore, with pore-scale imaging the flow and impact of surfactants and foams can be investigated. Future work must also focus on measuring three-phase relative permeabilities, which can be used to develop predictive models for three-phase flow in porous media.

Conventional laboratory measurements of three-phase relative permeability are prohibitively difficult, costly and time-consuming, and the reported results are often contradictory and misleading (Alizadeh and Piri 2014). This is attributed to the lack of understanding of the physical processes underlying three-phase flow in porous media. We hope that the insights provided in this paper can help interpret core-scale relative permeability measurements and promote the use of high-resolution imaging to understand the pore-scale physics of three-phase flow. We suggest that the use of pore-scale imaging with steadystate techniques, where both relative permeability and capillary pressure can be measured 
simultaneously, as applied in two-phase flow (Gao et al. 2019; Lin et al. 2018), can significantly improve the quality of three-phase relative permeability measurements.

Pore-scale imaging can provide solutions to some of the challenges often faced during steady-state three-phase relative permeability measurements. (i) In situ saturation measurement, which provides a direct assessment of the saturation profile in the pore space and can help identify and/or eliminate the regions influenced by boundary effects (e.g. capillary end effect), which are a major drawback of the steady-state approach. (ii) Direct assessment of wettability, which can be used to confirm the wettability of the sample to accurately interpret the results, especially to differentiate between weakly oil-wet and strongly oil-wet systems. (iii) Qualitative and quantitative assessment of pore occupancy and layer thickness.

Therefore, we recommend the development of an experimental approach that combines pore-scale imaging with steady-state flow to measure three-phase relative permeability. This will aid the development of rigorously validated, physically-based pore-scale models and empirical correlations to predict three-phase relative permeabilities under various wettability and miscibility conditions. The techniques could be adapted for several applications, including EOR, CCS and the design of microfluidic devices in food processing and drug delivery, for instance.

Funding We acknowledge Abu National Oil Company (ADNOC) for supporting this research.

Availability of Data and Materials Not applicable.

\section{Declarations}

Conflict of interest The authors have no conflicts of interest to declare that are relevant to the content of this article.

Open Access This article is licensed under a Creative Commons Attribution 4.0 International License, which permits use, sharing, adaptation, distribution and reproduction in any medium or format, as long as you give appropriate credit to the original author(s) and the source, provide a link to the Creative Commons licence, and indicate if changes were made. The images or other third party material in this article are included in the article's Creative Commons licence, unless indicated otherwise in a credit line to the material. If material is not included in the article's Creative Commons licence and your intended use is not permitted by statutory regulation or exceeds the permitted use, you will need to obtain permission directly from the copyright holder. To view a copy of this licence, visit http://creativecommons.org/licenses/by/4.0/.

\section{References}

Adamson, A.W., Gast, A.P.: Physical Chemistry of Surfaces. Interscience New York (1967)

Alhosani, A., Scanziani, A., Lin, Q., Pan, Z., Bijeljic, B., Blunt, M.J.: In situ pore-scale analysis of oil recovery during three-phase near-miscible $\mathrm{CO}_{2}$ injection in a water-wet carbonate rock. Adv. Water Resour. 134, 103432 (2019). https://doi.org/10.1016/j.advwatres.2019.103432

Alhosani, A., Scanziani, A., Lin, Q., Raeini, A.Q., Bijeljic, B., Blunt, M.J.: Pore-scale mechanisms of $\mathrm{CO}_{2}$ storage in oilfields. Sci. Rep. 10(1), 8534 (2020a). https://doi.org/10.1038/s41598-020-65416-Z

Alhosani, A., Scanziani, A., Lin, Q., Selem, A., Pan, Z., Blunt, M.J., Bijeljic, B.: Three-phase flow displacement dynamics and Haines jumps in a hydrophobic porous medium. Proc. R. Soc. A: Math. Phys. Eng. Sci. 476(2244), 20200671 (2020b). https://doi.org/10.1098/rspa.2020.0671

Alhosani, A., Lin, Q., Scanziani, A., Andrews, E., Zhang, K., Bijeljic, B., Blunt, M.J.: Pore-scale characterization of carbon dioxide storage at immiscible and near-miscible conditions in altered-wettability 
reservoir rocks. Int. J.Greenhouse Gas Control 105, 103232 (2021). https://doi.org/10.1016/j.ijggc. 2020.103232

Alizadeh, A.H., Piri, M.: Three-phase flow in porous media: a review of experimental studies on relative permeability. Rev. Geophys. 52(3), 468-521 (2014). https://doi.org/10.1002/2013RG000433

Allen, M., Antwi-Agyei, P., Aragon-Durand, F., Babiker, M., Bertoldi, P., Bind, M., Brown, S., Buckeridge, M., Camilloni, I., Cartwright, A. and Cramer, W.: Technical summary: global warming of $1.5^{\circ}$ C. An IPCC special report on the impacts of global warming of $1.5^{\circ} \mathrm{C}$ above pre-industrial levels and related global greenhouse gas emission pathways, in the context of strengthening the global response to the threat of climate change, sustainable development, and efforts to eradicate poverty (2019)

AlRatrout, A., Raeini, A.Q., Bijeljic, B., Blunt, M.J.: Automatic measurement of contact angle in pore-space images. Adv Water Resour. 109, 158-169, (2017). doi: https://doi.org/10.1016/j.advwatres.2017.07. 018

AlRatrout, A., Blunt, M.J., Bijeljic, B.: Wettability in complex porous materials, the mixed-wet state, and its relationship to surface roughness. Proc. Natl. Acad. Sci. 115(36), 8901 (2018). https://doi.org/10.1073/ pnas. 1803734115

Al-Siyabi, Z., Danesh, A., Tohidi, B., Todd, A.: Variation of gas-oil-solid contact angle with interfacial tension. Pet. Geosci. 5(1), 37-40 (1999)

Andrew, M., Bijeljic, B., Blunt, M.J.: Pore-scale imaging of trapped supercritical carbon dioxide in sandstones and carbonates. Int. J. Greenhouse Gas Control 22, 1-14 (2014). https://doi.org/10.1016/j.ijggc. 2013.12.018

Armstrong, R.T., Porter, M.L., Wildenschild, D.: Linking pore-scale interfacial curvature to column-scale capillary pressure. Adv. Water Resourc. 46, 55-62 (2012). https://doi.org/10.1016/j.advwatres.2012. 05.009

Bartell, F.E., Osterhof, H.J.: Determination of the wettability of a solid by a liquid: relation of adhesion tension to stability of color varnish and lacquer systems. Ind. Eng. Chem. 19(11), 1277-1280 (1927). https://doi.org/10.1021/ie50215a026

Bickle, M.J.: Geological carbon storage. Nature Geosci. 2(12), 815-818 (2009). https://doi.org/10.1038/ ngeo687

Blunt, M.J.: Constraints on contact angles for multiple phases in thermodynamic equilibrium. J. Colloid Interface Sci. 239(1), 281-282 (2001). https://doi.org/10.1006/jcis.2001.7534

Blunt, M.J.: Multiphase Flow in Permeable Media: A Pore-Scale Perspective. Cambridge University Press, Cambridge (2017)

Blunt, M., Fayers, F.J., Orr, F.M.P.: Carbon dioxide in enhanced oil recovery. Energy Convers. Manag. 34(9), 1197-1204 (1993). https://doi.org/10.1016/0196-8904(93)90069-M

Blunt, M.J., et al.: Pore-scale imaging and modelling. Adv. Water Resour. 51, 197-216 (2013). https://doi. org/10.1016/j.advwatres.2012.03.003

Blunt, M.J., Lin, Q., Akai, T., Bijeljic, B.: A thermodynamically consistent characterization of wettability in porous media using high-resolution imaging. J. Colloid Interface Sci. 552, 59-65 (2019). https://doi. org/10.1016/j.jcis.2019.05.026

Blunt, M.J., Alhosani, A., Lin, Q., Scanziani, A., Bijeljic, B.: Determination of contact angles for threephase flow in porous media using an energy balance. J. Colloid Interface Sci. 582, 283-290 (2021). https://doi.org/10.1016/j.jcis.2020.07.152

Brown, K., Schlüter, S., Sheppard, A., Wildenschild, D.: On the challenges of measuring interfacial characteristics of three-phase fluid flow with X-ray microtomography. J. Microscopy 253(3), 171-182 (2014). https://doi.org/10.1111/jmi.12106

Brownsort, P., Carruthers, K., Haszeldine, R.S., Johnson, G., Kapila, R., Kemp, A., Littlecott, C., Mabon, L., Mackay, E., Macrory, R. and Meyvis, B.: $\mathrm{CO}_{2}$ storage and enhanced oil recovery in the North Sea: securing a low-carbon future for the UK, SCCS (2015)

Bultreys, T., De Boever, W., Cnudde, V.: Imaging and image-based fluid transport modeling at the pore scale in geological materials: a practical introduction to the current state-of-the-art. Earth-Sci. Rev. 155, 93-128 (2016). https://doi.org/10.1016/j.earscirev.2016.02.001

Cassie, A., Baxter, S.: Wettability of porous surfaces. 40, 546-551 (1944)

Chen, Z., Xu, J., Wang, Y.: Gas-liquid-liquid multiphase flow in microfluidic systems: a review. Chem. Eng. Sci. 202, 1-14 (2019). https://doi.org/10.1016/j.ces.2019.03.016

Cnudde, V., Boone, M.N.: High-resolution X-ray computed tomography in geosciences: a review of the current technology and applications. Earth-Sci. Rev. 123, 1-17 (2013). https://doi.org/10.1016/j.earscirev. 2013.04.003

Falcone, G., Hewitt, G.F., Alimonti, C.: Multiphase flow metering. In: Developments in Petroleum Science, vol. 54, G. Falcone, G. F. Hewitt, and C. Alimonti Eds.: Elsevier, 2009, p. iii 
Fatemi, S.M., Sohrabi, M.: Recovery mechanisms and relative permeability for gas/oil systems at near-miscible conditions: effects of immobile water saturation, wettability, hysteresis, and permeability. Energy Fuels 27(5), 2376-2389 (2013). https://doi.org/10.1021/ef301059b

Feali, M., Pinczewski, W.V., Cinar, Y., Arn, C., Arns, J.Y., Francois, N., Turner, M., Senden, T., Knackstedt, M.: Qualitative and quantitative analyses of the three-phase distribution of oil, water, and gas in bentheimer sandstone by use of micro-CT imaging. SPE-151609-PA 15(06), 706-711 (2012). https://doi. org/10.2118/151609-PA

Fenwick, D.H., Blunt, M.J.: Network modeling of three-phase flow in porous media. In: SPE-60767-PA, 3(01): 86-96 (1998). doi: https://doi.org/10.2118/38881-PA

Ferrara, K.W., Borden, M.A., Zhang, H.: Lipid-shelled vehicles: engineering for ultrasound molecular imaging and drug delivery, (in eng). Acc. Chem. Res. 42(7), 881-892 (2009). https://doi.org/10.1021/ar800 2442

Gao, Y., Qaseminejad Raeini, A., Blunt, M.J., Bijeljic, B.: Pore occupancy, relative permeability and flow intermittency measurements using X-ray micro-tomography in a complex carbonate. Adv. Water Resour. 129, 56-69 (2019). https://doi.org/10.1016/j.advwatres.2019.04.007

Grate, J.W., et al.: Correlation of oil-water and air-water contact angles of diverse silanized surfaces and relationship to fluid interfacial tensions. Langmuir 28(18), 7182-7188 (2012). https://doi.org/10. 1021/la204322k

Heidug, W., Lipponen, J., McCoy, S., Benoit, P.: Storing $\mathrm{CO}_{2}$ through enhanced oil recovery: combining EOR with $\mathrm{CO}_{2}$ storage (EOR+) for profit. Insight Series (2015)

Hirasaki, G.J.: Structural interactions in the wetting and spreading of van der Waals fluids. J. Adhesion Sci. Technol. 7(3), 285-322 (1993). https://doi.org/10.1163/156856193X00718

Iglauer, S., Paluszny, A., Blunt, M.J.: Simultaneous oil recovery and residual gas storage: a pore-level analysis using in situ X-ray micro-tomography. Fuel 103, 905-914 (2013). https://doi.org/10. 1016/j.fuel.2012.06.094

Iglauer, S., Paluszny, A., Rahman, T., Zhang, Y., Wülling, W., Lebedev, M.: Residual trapping of $\mathrm{CO}_{2}$ in an oil-filled, oil-wet sandstone core: results of three-phase pore-scale imaging. Geophys. Res. Lett. 46(20), 11146-11154 (2019). https://doi.org/10.1029/2019GL083401

Kantzas, A., Chatzis, I., and F.A.L. Dullien. "Mechanisms of Capillary Displacement of Residual Oil by Gravity-Assisted Inert Gas Injection." Proceedings of the SPE Rocky Mountain Regional Meeting, SPE-17506-MS, Casper, Wyoming, May 1988. https://doi.org/10.2118/17506-MS

Kramer, D.: Negative carbon dioxide emissions. Phys. Today 73(1), 44-51 (2020)

Krevor, S., et al.: Capillary trapping for geologic carbon dioxide storage: from pore scale physics to field scale implications. Int. J. Greenhouse Gas Control 40, 221-237 (2015). https://doi.org/10.1016/j. ijggc.2015.04.006

Kundu, A., Dumont, E., Duquenne, A.M., Delmas, H.: Mass transfer characteristics in gas-liquid-liquid system. Can J Chem Eng 81, 640-646 (2008). https://doi.org/10.1002/cjce.5450810341

Lake, L.W.: Enhanced oil recovery. Elsevier (1989)

Lin, Q., Bijeljic, B., Pini, R., Blunt, M.J., Krevor, S.: Imaging and measurement of pore-scale interfacial curvature to determine capillary pressure simultaneously with relative permeability. Water Resour. Res. 54(9), 7046-7060 (2018). https://doi.org/10.1029/2018WR023214

Mayer, A.S., Miller, C.T.: An experimental investigation of pore-scale distributions of nonaqueous phase liquids at residual saturation. Transp. Porous Media 10(1), 57-80 (1993). https://doi.org/10.1007/ BF00617511

Melzer, S. Optimization of $\mathrm{CO}_{2}$ Storage in $\mathrm{CO}_{2}$ Enhanced Oil Recovery Projects. Advanced Resources International (2010)

Metz, B., Davidson, O., De Coninck, H., Loos, M., Meyer, L.: IPCC special report on carbon dioxide capture and storage. Intergovernmental Panel on Climate Change, Geneva (Switzerland). Working..., (2005)

Øren, P.E., Pinczewski, W.V.: The effect of wettability and spreading coefficients on the recovery of waterflood residual oil by miscible gasflooding. SPE-20185-PA, 9(02), 149-156 (1994). doi: https://doi.org/10.2118/24881-PA

Øren, P.E., Billiotte, J., Pinczewski, W.V.: Mobilization of waterflood residual oil by gas injection for water-wet conditions. SPE-20185-PA, 7(01), 70-78 (1992). https://doi.org/10.2118/20185-PA

Qi, R., LaForce, T.C., Blunt, M.J.: Design of carbon dioxide storage in oil fields. Proceedings of the SPE Annual Technical Conference and Exhibition, Denver, Colorado, USA (2008). [Online]. SPE115663-MS. Available: https://doi.org/10.2118/115663-MS.

Qin, Z., Arshadi, M., Piri, M.: Micro-scale experimental investigations of multiphase flow in oil-wet carbonates. II. Tertiary gas injection and WAG, Fuel, vol. 257, p. 116012, 2019/12/01/ 2019, https:// doi.org/10.1016/j.fuel.2019.116012 
Sabti, M., Alizadeh, A., Piri, M.: In-situ investigation of the impact of spreading on matrix-fracture interactions during three-phase flow in fractured porous media. Adv. Water Resour. 131 (2019). doi: https://doi.org/10.1016/j.advwatres.2019.05.017

Salathiel, R.A.: Oil recovery by surface film drainage in mixed-wettability rocks. SPE-949039-G 25(10), pp 1216-1224 (1973). doi: https://doi.org/10.2118/4104-PA

Sayed, M., Arshadi, M., Piri, M.: A micro-scale experimental investigation on the impact of varying stress on geo-mechanical deformation of proppant-packed fractured shale. Microsc. Microanal. 25(S2), 762-763 (2019). https://doi.org/10.1017/S1431927619004549

Scanziani, A., Singh, K., Bultreys, T., Bijeljic, B., Blunt, M.J.: In situ characterization of immiscible three-phase flow at the pore scale for a water-wet carbonate rock. Adv. Water Resour. 121, 446-455 (2018). https://doi.org/10.1016/j.advwatres.2018.09.010

Scanziani, A., Alhammadi, A., Bijeljic, B., Blunt, M.J.: Three-phase flow visualization and characterization for a mixed-wet carbonate rock. Proceedings of the Abu Dhabi International Petroleum Exhibition \& Conference, Abu Dhabi, UAE (2018). SPE-192666-MS [Online]. Available: https://doi. org/https://doi.org/10.2118/192666-MS

Scanziani, A., Singh, K., Menke, H., Bijeljic,, B., Blunt, M.J.: Dynamics of enhanced gas trapping applied to $\mathrm{CO}_{2}$ storage in the presence of oil using synchrotron X-ray micro tomography. Appl. Energy, 114136 (2019). doi: https://doi.org/10.1016/j.apenergy.2019.114136

Scanziani, A., et al.: In situ characterization of three-phase flow in mixed-wet porous media using synchrotron imaging. Water Resour. Res. 56(9), 873 (2020). https://doi.org/10.1029/2020WR027873

Scott, V., Gilfillan, S., Markusson, N., Chalmers, H., Haszeldine, R.S.: Last chance for carbon capture and storage. Nat. Climate Change 3(2), 105-111 (2013). https://doi.org/10.1038/nclimate1695

Sohrabi, M., Henderson, G.D., Tehrani, D.H., Danesh, A.: Visualisation of oil recovery by water alternating gas (WAG) injection using high pressure micromodels - water-wet system. Proceedings of the SPE Annual Technical Conference and Exhibition, Dallas, Texas, 2000/1/1/, 2000. SPE-63000-MS [Online]. Available: https://doi.org/https://doi.org/10.2118/63000-MS

Sohrabi, M., Tehrani, D.H., Danesh, A., Henderson, G.D.: Visualisation of oil recovery by water alternating gas (WAG) injection using high pressure micromodels - oil-wet \& mixed-wet systems. Proceedings of the SPE Annual Technical Conference and Exhibition, New Orleans, Louisiana, 2001/1/1/, 2001. SPE71494-MS [Online]. Available: https://doi.org/https://doi.org/10.2118/71494-MS

Sohrabi, M., Tehrani, D.H., Danesh, A., Henderson, G. D.: Visualization of oil recovery by water-alternating-gas injection using high-pressure micromodels. SPE-60767-PA, vol. 9, no. 03, pp. 290-301 (2004). doi: https://doi.org/10.2118/89000-PA

Sohrabi, M., Danesh, A., Tehrani, D.H., Jamiolahmady, M.: Microscopic mechanisms of oil recovery by near-miscible gas injection. Transp. Porous Media 72(3), 351-367 (2008). https://doi.org/10.1007/ s11242-007-9154-Z

Sorbie, K.S., van Dijke, M.I.: The mechanism of oil recovery by water-alternating-gas injection at nearmiscible conditions in mixed wet systems (2010). https://doi.org/https://doi.org/10.2118/129837-MS

Stone, H.L.: Probability model for estimating three-phase relative permeability. SPE-949039-G, 22(02), 214-218 (1970). doi: https://doi.org/10.2118/2116-PA

Stone, H.L.: Estimation of three-phase relative permeability and residual oil data. PETSOC-73-04-06, vol. 12, no. 04, p. 10 (1973). doi: https://doi.org/10.2118/73-04-06.

van Dijke, M.I.J., Sorbie, K.S.: An analysis of three-phase pore occupancies and relative permeabilities in porous media with variable wettability. Transp. Porous Media 48(2), 159-185, (2002). doi: https://doi. org/10.1023/A:1015692630733

van Dijke, M.I.J., Sorbie, K.S.: Pore-scale modelling of three-phase flow in mixed-wet porous media: multiple displacement chains. J. Petrol. Sci. Eng. 39(3), 201-216 (2003). doi: https://doi.org/10.1016/ S0920-4105(03)00063-9

Vizika, O., Lombard, J.M.: Wettability and spreading: two key parameters in oil recovery with three-phase gravity drainage. In: SPE-25254-PA, 11(01): 54-60 (1996). doi: https://doi.org/10.2118/28613-PA

Wenzel, R.N.: Resistance of solid surfaces to wetting by water. Ind. Eng. Chem. 28(8), 988-994 (1936). https://doi.org/10.1021/ie50320a024

Wildenschild, D., Sheppard, A.P.: X-ray imaging and analysis techniques for quantifying pore-scale structure and processes in subsurface porous medium systems. Adv. Water Resour. 51, 217-246 (2013). https://doi.org/10.1016/j.advwatres.2012.07.018

Zhao, C.-X.: Multiphase flow microfluidics for the production of single or multiple emulsions for drug delivery. Adv. Drug Deliv. Rev. 65 (2013). doi: https://doi.org/10.1016/j.addr.2013.05.009

Zúñiga, R.N., Aguilera, J.M.: Aerated food gels: fabrication and potential applications. Trends Food Sci. Technol. 19(4), 176-187 (2008). https://doi.org/10.1016/j.tifs.2007.11.012 
Publisher's Note Springer Nature remains neutral with regard to jurisdictional claims in published maps and institutional affiliations.

\section{Authors and Affiliations}

\section{Abdulla Alhosani $^{1}$ (D) Branko Bijeljic $^{1} \cdot$ Martin J. Blunt $^{1,2}$ (D)}

$\triangle$ Abdulla Alhosani

Abdulla.alhosani17@imperial.ac.uk

1 Department of Earth Science and Engineering, Imperial College London, London, UK

2 Department of Energy, Politecnico di Milano, Milan, Italy 